\title{
Market-based Network Formation for an Ad Hoc, P2P Wireless Network
}

\author{
Yasunori Yamamoto* \\ School of Information Studies, Syracuse University \\ Ministry of Public Management, Home Affairs, Posts and Telecommunications \\ 5435-1-502 Ooyaguchi, Saitama 336-0934 JAPAN \\ yayamamo@syr.edu
}

\author{
Junseok Hwang ${ }^{\dagger}$ \\ School of Information Studies, Syracuse University \\ 4-291, Center for Science and Technology \\ Syracuse, NY 13244-4100 USA \\ jshwang@syr.edu
}

Abstract In an ad hoc, arbitrarily formed peer to peer (P2P) network system, each user can select one of the services offered by multiple neighbor stations to have an access to the network. Every user has a preference function to determine a service to use, which is described as a total willingness to pay based on the bandwidth and the duration of using a service. In our study, we developed a market-based model for a user to determine a service in order to maximize the user's surplus. Our model allows a station to connect another station without reconnection as long as it wants or possible. By employing our model, stations of providing a service can offer a competitive pricing based on durations of connections such as a discount for their users to use it longer. The pricing of this kind is also preferable for both users and providers. In addition, based on our model, we developed an algorithm for a station to determine a way of making a connection to the network. We simulated some scenarios of ad hoc P2P wireless networks by using the algorithm. We found that the formed network is more efficient than former ceaselessly reconnecting networks in terms of the connectivity to get a certain QoS.

Keywords: Market-based networks, P2P networking, Wireless Communications

\footnotetext{
*Phone:+81(48)874-7233, Fax:+1(702)921-3399

tPhone:+1(315)443-4473, Fax:+1(315)443-5806
}

The original version of this chapter was revised: The copyright line was incorrect. This has been corrected. The Erratum to this chapter is available at DOI: 10.1007/978-0-387-35618-1_37 


\section{Introduction}

Due to rapid development of the wireless technology, demands on high-speed personal wireless data communications has increased (International Telecommunication Union, 2000). Once in the range of propagation, individual users can access the Internet without any cable wherever they are, whether they move or not. In this situation, decentralized ad hoc P2P networks are more suitable than centralized static network systems. Those networks allow every station to become a network provider for other stations and act as a repeater, so that a user out of the range of a base station could access the network via another station. We assumed the following scenario. In an ad hoc, arbitrarily formed P2P network system, each user selects one of the services offered by multiple neighbor stations to have an access to the network. Every user has a preference function to determine a service to use, which is described as a total willingness to pay based on the bandwidth and the duration of using a service. In our study, we developed a market-based model for a user to determine a service in order to maximize the user's surplus. In our model, a station can connect with another without a reconnection as long as it wants or possible. This feature allows stations to provide a competitive pricing such as a discount plan for their users to connect with them longer. In order to realize it, users are previously informed of costs according to the duration of a connection by providing stations. The pricing scheme of this kind brings benefits to both of users and providers, since many users prefer a fixed rate service to that of usage-based charging (Chu, 1999), while providers can get an optimal pricing to make their profits higher. In spite of many studies of optimal pricing, few of them take consideration of this users' preference.

In addition, based on the market-based model, we developed an algorithm of determining a way of connecting with the network. We simulated some scenarios of ad hoc P2P wireless network formations by using the algorithm. As a result, we found that an ad hoc P2P wireless network can be formed autonomously by using the algorithm. The formed network showed better performance than ceaselessly reconnecting networks in terms of the connectivity.

\section{Related Studies}

Some studies proposed a bidding method to allocate resource (i.e., a seller allocates resource to those who value it most) and used a game theory to show an optimal condition under which users and a seller maximize their utilities. Users bid every certain period of time to be allocated. A recent study (Lazar and Semret, 1998) introduced the Pro- 
gressive Second Price (PSP) mechanism, which is derived from Vickrey Auction (second price sealed bid) and generalizes it. Resource is allocated to bidders according to the order of the bidding prices until no more resource is left. This mechanism is unprecedented in that it applies to a generic arbitrarily divisible and additive resource model such as spectrum allocation. Their approach does not assume any specific mapping of resource allocation to quality of service. However, bidders are defined as having an explicit monetary valuation of quantities of resource which auctioneer does not or cannot know a priori.

Another approach (Altmann et al., 2002) used a Markov model. In the model, an administrator offers several types of services at different priority levels, and a user decides one of them to use based on his or her job's priority and offered prices. A price at a priority level does not change but an actual QoS at the level varies depending on circumstances. Accordingly, users can see a QoS and its price of a service when deciding. Since this approach is job oriented, however, users cannot estimate total costs for their connections.

\section{Assumption}

We consider two main types of network topology when we develop a pricing model: Star and P2P. Star topology is that there is an access point used by multiple users simultaneously. Current cellular system is in this topology. P2P topology is that there are multiple stations and a station can be an access point to the others. Needless to say, this topology includes Star topology. An advantage of this can be shown when there is a station out of the range of an access point, but another station within the range can become a proxy to that station.

Since we assume that any user can also be a network provider to another, we do not care about a network topology but focus on behaviors of users and providers. Users prefer fixed rate services while providers seek maximum profits. We seek a way of providing a condition under which both players can get satisfied. A provider offers several types of services in terms of duration of service and bandwidth (QoS). A user chooses a service offered by neighbor providers according to his or her preference.

We assume a CDMA system to which our model applies, where users are assigned orthogonal spreading codes. In this system, a transmitted power and a length of codes determine a QoS. As in a study (Liu et al., 2000), we assume all codes have same length, and therefore a QoS is determined by a transmitted power. Let $\mu_{i}\left(0<\mu_{i}<1\right), d_{i}\left(1 \leq d_{i}\right)$, and $P_{j}$ represent the channel gain of user $i$, the distance between $i$ and 
the transmitter, and the allocated power of service $j$ for user $i$ at the distance of the one unit length from the transmitter, respectively. It should be noted that we ignore such propagation factors as shadowing and multipath. Since received power can be determined by the channel gain and the distance from the transmitter $d_{i}$ and the path loss exponent $\kappa, \mu_{i} d_{i}^{-\kappa} P_{j}$ is the average received power at user $i$ with service $j$. In addition, an average transmission rate at user $i$ is determined by the received power, which is $s_{j i}=\zeta_{i} d_{i}^{-\kappa} P_{j}\left(\zeta_{i} \propto \mu_{i}\right)$. Assuming that a provider sends data to user $i$ with an average transmission rate $s_{j}$ at the point of the provider, once a QoS of service $j$ and the distance $d$ is determined, its transmitted power can be settled. In this situation, a provider offers a user its service plans which consist of pricing and discount schedules. Those pricings are based on bandwidths (i.e., $s_{j}$ ) and service durations. Then, the user decides to use one of them, or otherwise gives up.

\section{Model}

Utility function. Utility function $u_{i}$ which determines the surplus for a user $i \in\{1, \cdots, I\}$ is:

$$
u_{i}=t w_{i}\left(v, s_{j i}\right)-c_{j}\left(v, s_{j}\right)
$$

while $t w_{i}$ is a user $i$ 's total willingness to pay, $c_{j}$ is the cost to use a service $j \in 1, \cdots, N$, and $v \in[0, T]$ is the duration of service. A service provider does not determine a service price depending on bandwidth but on output power to meet designated bandwidth at the transmitter.

Let $b \in[0, \infty)$ represent the bandwidth. Since we assume that both of user $i$ 's preferences (i.e., service duration and bandwidth) need to be satisfied when accepting an offer, total willingness to pay can be:

$$
t w_{i}(v, b)=\alpha t w_{i v}(v) t w_{i b}(b)
$$

where $\alpha$ is $i$ 's budget, $t w_{i v}(v) \in[0,1]$ and $t w_{i b}(b) \in[0,1]$ are the normalized $i$ 's total willingness to pay functions of service duration and of bandwidth, respectively. The reason why we introduce the factor of the service duration is that the individual users prefer fixed rate services, and in other words, pricing plans for a certain bandwidth or power can be described as the service duration and a discount rate at the duration time. Therefore,

$$
u_{i}=t w_{i}\left(v, \zeta d_{i}^{-\kappa} P_{j}\right)-c_{j}\left[k_{j}(v), \int_{t}^{(t+v)} \phi_{j}(\tau) d \tau\right]
$$

where $k_{j}(v) \in[0,1]$ is the discount rate of making a service $j$ with a duration $v$, and $\phi_{j}(t)$ is the price of making a service $j$ connection for one unit of time at time $t$. 
Pricing Scenario. As in a study (Wang et al., 1996), the service duration is exponentially distributed with mean $1 / r_{j}$ for any service $j$, where $r_{j}$ is the rate of service happening. We also assume that a provider gives a pricing and a discount schedule represented as $\left[\phi_{1}(t), \phi_{2}(t), \cdots, \phi_{N}(t)\right]$ and $\left[k_{1}(d), k_{2}(d), k_{N}(d)\right]$, respectively. Assuming that the exponentially distributed service duration is not affected by $\phi_{j}(t)$, users starting a service $j$ at time $t$ are expected to pay an average price of:

$$
p_{j}(t)=\int_{t}^{+\infty}\left[r_{j} e^{-r_{j}(\tau-t)} \cdot \int_{t}^{(\tau-t)} \phi_{j}(\nu) d \nu \cdot\left(1-k_{j}(\tau-t)\right)\right] d \tau .
$$

Let $p_{b}(t)$ be a spot price determined by how scarce the spectrum resource is at time $t$. $p_{b}(t)$ would become higher if, for example, the propagation condition is worse or number of users wanting to use it increases. Since $\phi_{j}(t)$ can be uniquely determined by $p_{b}(t)$ if the discount rates are given, we assume that providers manipulate $\left[p_{1}(t), p_{2}(t), \cdots, p_{N}(t)\right]$ to maximize their utility.

\section{Case Study}

\subsection{Station's Behavior (With vs. Without a Discount)}

As an example of comparison, we took three service plans $\phi_{i}(i=1,2$, and 3) with and without a discount plan $k_{1}{ }^{1}$. In addition, we took a user's preference $t w_{1}$ for those services as an example, which can be described as a Gaussian function. In this situation, the user's surplus $U(t)$ can be described as follows:

$$
U(t)=t w_{1}(t)-k_{1}(t) \int_{0}^{t} \phi_{i}(\nu) d \nu(i=1,2,3) .
$$

Therefore, in this example, the duration of time to make the user's surplus optimal can be found by solving the following equation:

$$
\frac{d t w_{1}(t)}{d t}=\frac{d}{d t} k_{1}(t) \int_{0}^{t} \phi_{i}(\nu) d \nu(i=1,2,3) .
$$

s.t.

$$
t w_{1}>k_{1}(t) \int_{0}^{t} \phi_{i}(\nu) d \nu
$$

Figure 1 shows how these three pricing plans require a user to pay according to the duration of connection time, and a user's preference.

${ }^{1} k_{1}(t)=1$ in case of no discount. 


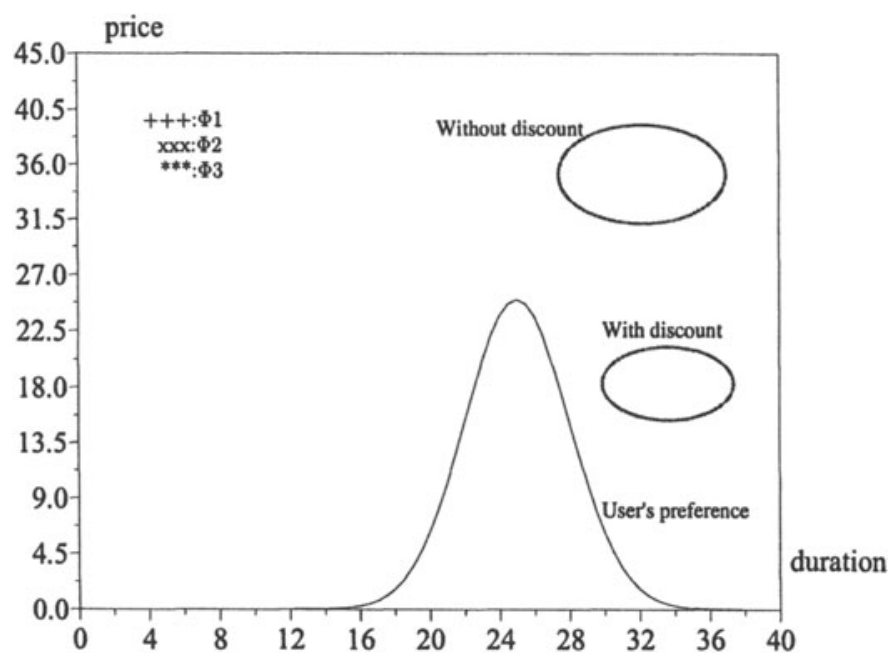

Figure 1. Comparison of services, and a user's preference

\subsection{Network Formation}

Algorithm. In order to make a network formation autonomic, we developed an algorithm for a station to determine a way of establishing a connection to another station or giving up. Table 1 shows the algorithm, which employs our model discussed in this study.

Table 1. Algorithm to make a connection

$P=\{\}, Q=\{\}$

For $i=1$ To the end of existing stations nearby

Calculate the distance $d_{i}$;

For $j=1$ To the end of services which station $i$ offers

$Q=Q \cup\left\{Q o S\right.$ of Service $\left.s_{i j}\right\}$

$P=P \cup\left\{\right.$ Pricing Schedule of Service $\left.s_{i j}\right\}$

End;

End;

Find a service $s_{i^{*} j^{*}}$ :surplus $s p\left(s_{i^{*} j^{*}}\right)>s p\left(s_{i j}\right)\left(i^{*} \neq i, j^{*} \neq j\right)$;

If $s_{i^{*} j^{*}}<0$ Then

Give up making a connection;

Else Make a connection to get the service $s_{i^{*} j^{*}}$;

End; 
Discrete vs. Continuative Connection. In order to evaluate our model, we simulated ad hoc P2P network formations in two situations. One set of simulations employed our algorithm which enables a station to have a continuative connection to another over a period of time. The other scenario also employed our algorithm but continuative connections were disabled. All conditions were identical except for the difference in the continuation. In our simulation, 360 stations were generated according to Poisson distribution. Life spans of them also followed the distribution. Collected data from each simulation include: a total times to complete $e^{2}$, average numbers of active ${ }^{3}$ and inactive $e^{4}$ stations, and an average depth ${ }^{5}$ of formed networks. Figure 2 and 3 show the difference between continuative and discrete connections. Both figures show the

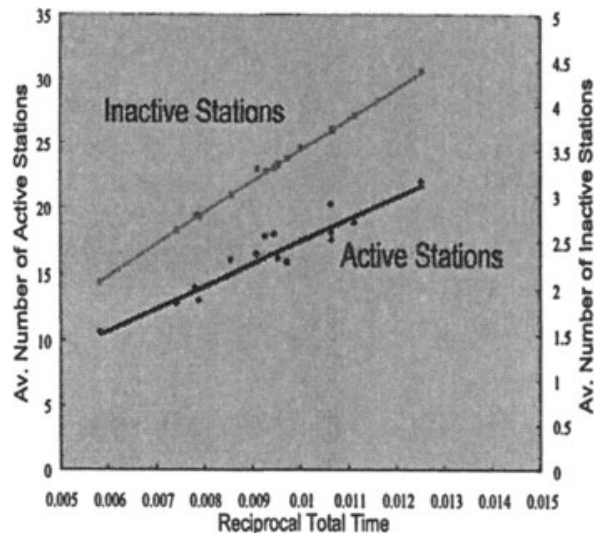

Figure 2. Discrete Connections

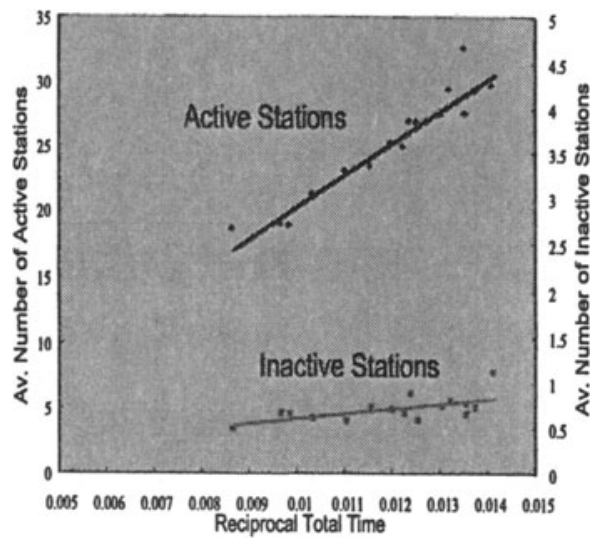

Figure 3. Continuative Connections

Average Number of Active and Inactive Stations

average numbers of active and inactive stations to the reciprocal total times to complete a simulation. As for the discrete connections, the average numbers of active stations are from 10.6 to 22.1 while those of inactive ones are from 2.06 to 4.40 . On the other hand, concerning the continuative connections, those of active ones are from 18.6 to 32.5 and inactive ones from 0.50 to 1.12 . This result indicates that more stations can have connections when they are continuative than discrete. In other words, the connectivity of an ad hoc P2P network improves when con-

\footnotetext{
${ }^{2}$ from the time of the first station comes up to the time of the last one disappears

3 station having a connection

4 station not having a connection

${ }^{5}$ number of connections from the root to a station (e.g., a star topology network has 1.00 of its average depth.)
} 
tinuative connections are allowed. It should be noted that the average depths of both situations are almost identical (1.73 and 1.84).

\section{Conclusion}

In our study, we introduce a discount factor, and therefore fixed rate services which many individual users' preference can be described in our pricing model. Each user is previously shown with service plans which comprise a pricing and a discount schedule by multiple service providers. After that, the user can determine a service plan and a service provider to use. Besides, we do not assume that each service provider previously knows the users' preferences, but instead assume that their durations of time to use networks are exponentially distributed. In this assumption, we get an optimal pricing model.

In addition, we developed an algorithm for each station to determine how to make a connection to another station based on the surplus for it. Using the algorithm, we made simulations and confirmed that an ad hoc P2P wireless network can be formed autonomously.

In addition, our simulations showed that connectivity of formed networks improves by introducing continuative connections.

\section{References}

Altmann, J., Daanen, H., Oliver, H., and Suárez, A. S.-B. (2002). How to MarketManage a QoS network. In IEEE InfoCom 2002, Conference on Computer Communications, New York, USA.

Chu, K. (1999). User reactions to flat rate options under time charges with differentiated quality of access: Preliminary results from index.

International Telecommunication Union (2000). Main results of WRC-2000. Retrieved September 26, 2001, from: http://www.itu.int/brconf/wrc-2000/docs/index.html.

Lazar, A. A. and Semret, N. (1998). Design and analysis of the progressive second price auction for network bandwidth sharing. The 8th International Symposium on Dynamic Games and Applications.

Liu, P., Honig, M. L., and Jordan, S. (2000). Forward-link CDMA resource allocation based on pricing. In IEEE Wireless Communications and Networking Conference, Chicago, IL.

Wang, Q., Sirbu, M. A., and Peha, J. M. (1996). Telecommunications and Internet Policy, chapter Pricing of ATM Network Services. Lawrence Erlbaum Assoc, Washington, DC. 